\title{
INVESTIGATION OF SILICON WAFERS' INFLUENCE ON THE LOCAL MICROWAVE POWER VALUES IN A RESONATOR-TYPE PLASMATRON
}

\author{
S. Madveika, S. Bordusau*, M. Lushakova, O. Tsikhan \\ Belarusian State University of Informatics and Radioelectronics, P.Brovki 6, 220013 Minsk, Belarus \\ * bordusov@bsuir.by
}

\begin{abstract}
The article concerns the investigation results of the $\varnothing 100 \mathrm{~mm}$ silicon wafers' influence on the microwave power value $\left(f_{\mathrm{MW}}=2.45 \pm 0.05 \mathrm{GHz}\right)$ in local points on the axis of a reaction-discharge chamber with the volume of about $9000 \mathrm{~cm}^{3}$ of a resonator-type plasmatron. The experiments were carried out in the conditions of the dynamic microwave power redistribution inside a volumetric resonator by using a moving dissector. To register microwave power in the plasma volume, the method of "the active probe" was used. It has been experimentally established that the decrease of distance between the silicon wafers results in the decrease of local microwave power values between them up to $50 \%$. The investigation results of the silicon wafers' influence on the microwave power distribution structure in the gas discharge area indicate the presence of power distribution nonuniformity in the discharge area volume.
\end{abstract}

Keywords: microwave plasma, silicon wafers, microwave power, plasmatron.

\section{Introduction}

New tasks and high micro- and nanoelectronics' production requirements stimulate the search for new and modernization of existing methods of a controlled plasma impact on the treated materials. Nowadays there is a great interest in the industrial application of a ultra-high-frequency (UHF) discharge, the socalled microwave discharge or microwave plasma, in the processes of electronic devices production.

The advantage of UHF range is the possibility to create a discharge device construction for excitation of plasma with a vast spectrum of parameters [1-3]. The technical methods used for excitation of microwave plasma are characteristic just for a UHF range and differ from those used at other frequencies. This is due to the fact that the wavelength is comparable with the size of the discharge device, and for their design systems with distributed parameters (waveguides, resonators, etc.) are often used.

A microwave discharge with a localized discharge region (resonator and waveguide type of discharges) has certain advantages in the solution of the problem of a plasma spatial structure influence on its chemical activity [4].

Non-equilibrium plasma of microwave discharges makes it possible to carry out plasma-chemical processes at a low gas temperature but at a higher electron temperature [5].

In a microwave discharge plasma, when the electrodynamic characteristics of a microwave applicator are changed, it is possible to control the internal discharge structure [6], which is impossible, for example, in a direct current discharge. From this point of view, volume resonators are perspective technical solutions for localization of microwave energy in a plasma volume.

However, in resonator-type plasma systems in case of high energy inputs into plasma, a large plasma non-uniformity can be formed because of significant electromagnetic field intensity gradients. It imposes certain limits on the processes of plasma-chemical treatment of semiconductor wafers with a diameter of more than $200 \mathrm{~mm}$ and in group treatment of smaller diameter wafers [7].

In order to reduce the microwave power nonuniformity in the plasma volume of resonator-type plasmatrons, microwave power redistribution systems are used [1]. The simplest technical solution is a rotating metal dissector placed in the resonator [8]. However, there is no information about the influence of silicon wafers with a high dielectric loss tangent on the local microwave power values in resonator-type plasmatrons with microwave power redistribution using a moving dissector.

Therefore, the investigation of the influence of the objects of treatment (their material, volume, shape, size, etc.) on the electrophysical and chemical properties of plasma as well as technological processes of treating materials with microwave plasma are of great interest.

\section{Experimental apparatus and procedures}

The investigation of the influence of silicon wafers with a high dielectric loss tangent on the local microwave power values during its dynamic redistribution in the microwave plasma was carried out in a microwave resonator-type plasma installation [9].

The principle of operation of the experimental vacuum-plasma installation is based on the transfer of microwave power from a microwave magnetron M-105 $\left(f_{\mathrm{MW}}=2.45 \pm 0.05 \mathrm{GHz}\right)$ into an evacuated reaction- 
a)

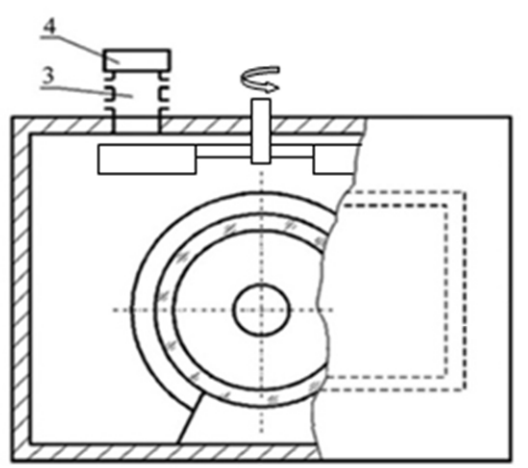

b)

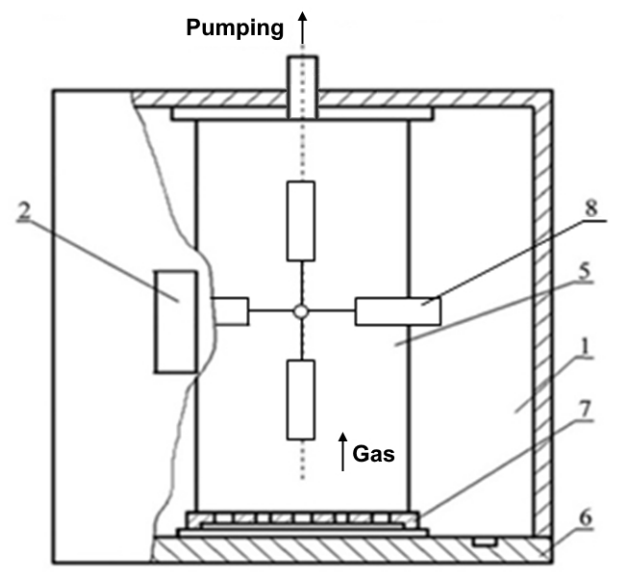

Figure 1. Scheme of the reaction-discharge system of the microwave plasma installation ( a - front view; $b-$ top view):

1 - resonator; 2 - coupling aperture; 3 - waveguide; 4 - magnetron; 5 - reaction-discharge chamber; 6 resonator's front wall; 7 - chamber's front cover; 8 - dissector.

discharge chamber (RDC) where a low temperature gas microwave discharge is excited by a UHF electromagnetic field. Bottled $99.7 \%$ oxygen was as used as a plasma-forming gas. The operation pressure in the chamber equalled to $130 \mathrm{~Pa}$.

The reaction-discharge chamber is made of quartz tube of $20 \mathrm{~cm}$ in diameter and $34 \mathrm{~cm}$ long having a total volume of about $9000 \mathrm{~cm}^{3}$ and is placed in the center of a $40 \times 40 \times 30 \mathrm{~cm}^{3}$ volume resonator (see figure 1). The electromagnetic energy from the microwave magnetron is fed into the resonator through a rectangular coupling apperture in the top wall of the resonator. A metal L-form dissector is attached to the top wall inside the resonator. The dissector is placed in such a way that when it revolves its blades cross the space under the coupling apperture. The microwave magnetron in the installation worked in the pulse mode in the conditions of generating a nominal power of $P_{\mathrm{MW}} \approx 650 \mathrm{~W}$.

The microwave power measurements at the local points on the axis of the discharge chamber with plasma and without it were performed using the "active probe" method $[5,8]$. The "active probe" is a seg- ment of a flexible coaxial cable with a wave impedance of $50 \Omega$, the external conductor of which is a copper pipe and the internal one is a single-core copper wire. The space between the conductors is filled with a flexible dielectric - teflon. An "active probe", which is a segment of the central internal conductor with a diameter of $1.4 \mathrm{~mm}$ protruding from the external conductor by $5 \mathrm{~mm}$, is formed on one side of the cable. The indications of the "active probe" were registered with a microwave power meter M3-51.

Silicon wafers with a diameter of 76 and $100 \mathrm{~mm}$ $(\rho=1.11 \Omega \cdot \mathrm{cm})$ were used in the research. In each series of experiments in the discharge chamber there were two wafers of the same size placed along the gas flow parallel to each other, one on each side of the probe symmetrical to it. The distance between the wafers varied within the range of $20-100 \mathrm{~mm}$.

In the first series of experiments the influence of silicon wafers on the overall structure of the microwave power distribution in plasma along the axis of the discharge chamber was studied. For this purpose the probe was moved along the discharge chamber. The investigation was performed using two silicon wafers with a diameter of $100 \mathrm{~mm}$. The distance between the wafers equaled to $60 \mathrm{~mm}$. The wafers were placed in the central part of the discharge chamber. In some local areas the probe passed between the silicon wafers. The indications of the "active probe" were registered along the axis every $20 \mathrm{~mm}$ in the direction from the chamber's front cover.

In the second series of experiments the influence of the distance between the silicon wafers (in the range of $20-100 \mathrm{~mm}$ ) on the local values of microwave power between them was examined. The experiments were performed with silicon wafers with a diameter of 76 and $100 \mathrm{~mm}$ placed in the central part of the discharge chamber. The "active probe" was placed between the wafers motionless.

\section{Results and discussion}

Figure 2 shows the experimental values of microwave power meter indications in the plasma volume along the axis of the discharge chamber without wafers (figure 2a) and with two silicon wafers with a diameter of $100 \mathrm{~mm}$ (figure 2b), placed in the central part of the discharge chamber.

As it can be seen from the data presented in figure 2 the value of the microwave power between the silicon wafers decreases by approximately $20-30 \%$. The changes of probe's indications in the plasma chamber's areas close to the location of silicon wafers are insignificant. At the same time, the general structure of the electromagnetic energy distribution in plasma in the microwave plasmatron's discharge chamber is maintained. The periodicity of alternations of maximum and minimum probe's indications corresponds to a half-length $\left(f_{\mathrm{MW}}=2.45 \pm 0.05 \mathrm{GHz}\right)$ of the electromagnetic wave exciting the plasma. 


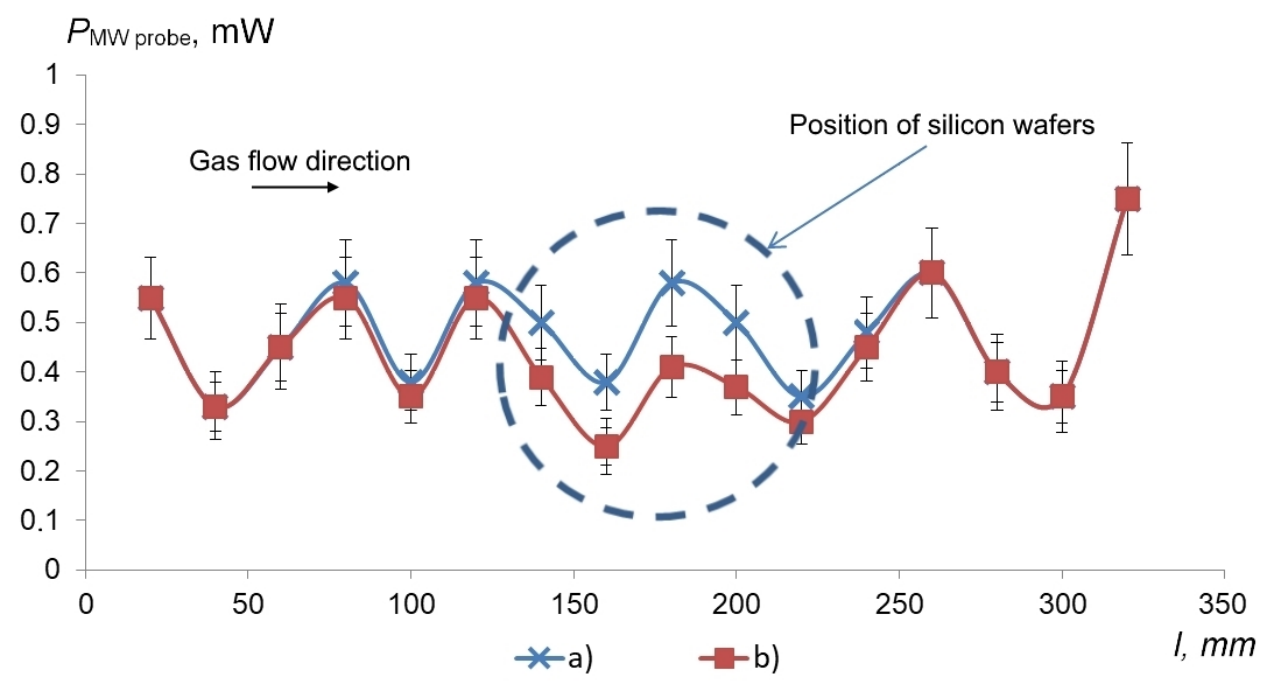

Figure 2. Experimental values of microwave power meter indications in plasma along the axis of the discharge chamber: $a$ - without wafers; $b$ - with two silicon wafers with a diameter of $100 \mathrm{~mm}$ placed in the central part of the discharge chamber.

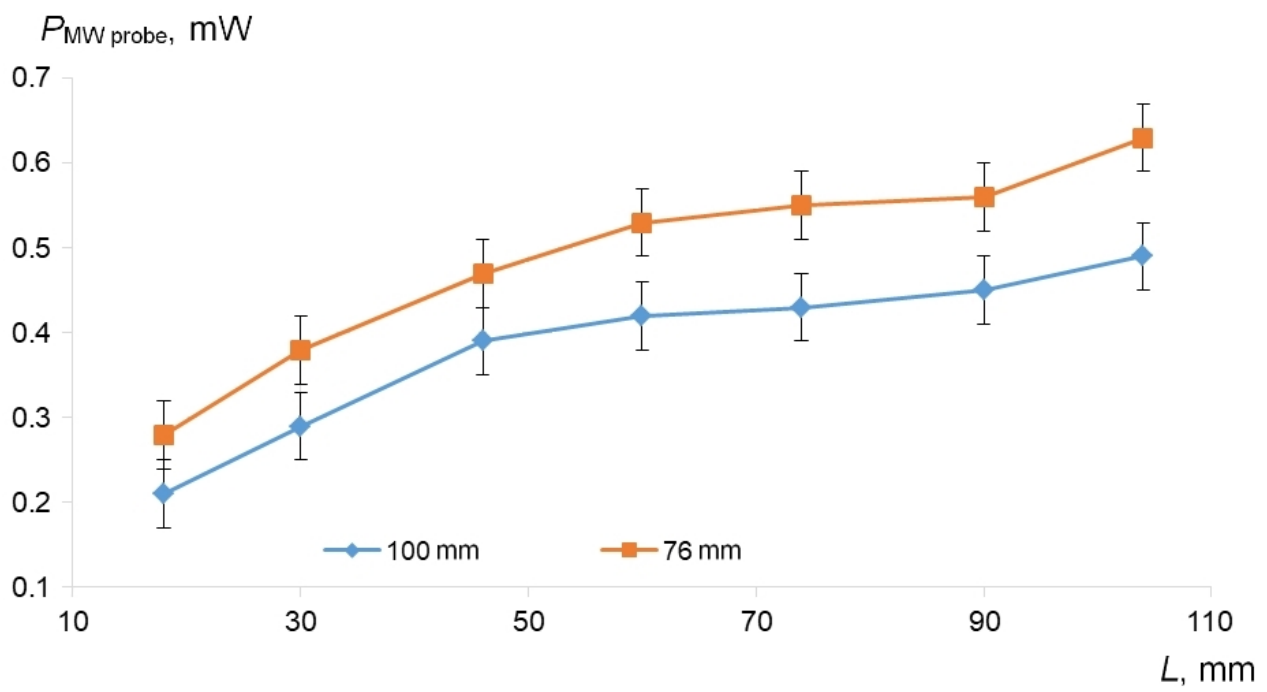

Figure 3. Dependence of microwave power value in the plasma volume on the distance between two silicon wafers with diameters of 76 and $100 \mathrm{~mm}$.

Figure 3 shows the diagrams of the experimental dependencies of the microwave power meter's indications in the plasma volume on the distance $L$ between the pairs of silicon wafers of 76 and $100 \mathrm{~mm}$ in diameter.

From the dependencies presented in figure 3 it can be seen that the change of the distance between silicon wafers from $100 \mathrm{~mm}$ to $20 \mathrm{~mm}$ results in the approximate $50 \%$ decrease of the microwave power between the wafers both for the pair of wafers with $100 \mathrm{~mm}$ in diameter as well as for the wafers with $76 \mathrm{~mm}$ in diameter. The behavior of dependencies for silicon wafers of both diameters is of the same character. The presented data also show that the increase of silicon wafers' diameter results in the decrease of local microwave power values between them.

It is assumed that the effects observed in both series of experiments can be explained by partial absorption and shielding of microwave radiation by silicon wafers having high dielectric loss tangent.

Thus, the change in the position of the silicon wafers in the reaction-discharge chamber of the microwave resonator-type plasmatron, their size and mutual arrangement significantly affect the value of the microwave energy penetrating into the plasma volume.

This should be taken into account in the analysis of plasma forming processes in the conditions of microwave plasma-chemical treatment with a volumetric positioning of materials in the microwave plasmatrons' discharge chambers.

\section{Conclusion}

The performed research has shown that the insertion of silicon wafers into the discharge chamber of a microwave resonator-type plasmatron does not significantly alter the overall structure of non-uniform 
microwave power distribution in the volume of plasmaforming zone. In this case the spatial alternation periodicity of its maxima and minima in plasma remains the same as in the case without wafers.

It has been experimentally established that with the increase of silicon wafers' diameter the value of microwave power between them decreases. The change in the distance between the silicon wafers also results in the change of the microwave power value between them.

The performed experiments allow to supplement the model conception of physico-chemical peculiarities of microwave plasma-chemical treatment processes of materials. Particularly it concerns the effect of influence of silicon wafers on the local microwave power values in the plasma chamber volume which can also affect the degree of chemical activity of different gas discharge areas. This effect should be taken into account while designing the constructions of microwave discharge systems for technological purposes and processes of group microwave plasma-chemical treatment of electronic devices that use monocrystalline silicon wafers as substrates.

The obtained results may be used in designing of new gas discharge systems and the modernization of existing technological equipment for a group nonprecision microwave plasma-chemical treatment of semiconductor wafers.

\section{Acknowledgements}

This research has been supported by the State Program of Scientific Research "Physical materials science, new materials and technologies", assignment 3.5.08.

\section{References}

[1] S. Bordusov. Microwave plasma technologies in the production of electronic devices. Minsk: Bestprint, 2002.

[2] Y. Lebedev. Microwave discharges at low pressures and peculiarities of the processes in strongly non-uniform plasma. Plasma Sources Science and Technology, 24(5):139, 2015. doi:10.1088/0963-0252/24/5/053001.

[3] V. Batenin, I. Klimovsky, G. Lisov, and V. Troitski. Microwave Plasma Generators: Physics, Engineering, Applications. Moscow: EnergoAtomlzdat, 1988.

[4] Y. Lebedev. The chemical activity of nonequilibrium plasma. state and perspectives of research. Chemical Physics, 15(5):96-100, 1996.

[5] U. Kortshagen. Electron and ion distribution functions in RF and microwave plasmas. Plasma Sources Science and Technology, 4(4):172-182, 1995. doi:10.1088/0963-0252/4/2/002.

[6] T. Nakano and S. Samukawa. The correlation between an electric field and the metastable chlorine ion density distributions in an ultrahigh-frequency plasma. Japanese Journal of Applied Physics, 37(5R):2686-2692, 1998. doi:10.1143/jjap.37.2686.

[7] A. Didenko and B. Zverev. Microwave energetics. M.: Nauka, 2000.

[8] S. Bordusau, S. Madveika, and A. Dostanko. Investigation of microwave energy distribution character in a resonator type plasmatron. Plasma Physics and Technology, 3(3):122-125, 2016.

[9] S. Bordusau. Design features of the installation and processes of microwave plasma materials processing under low vacuum. Materials, technology, instruments, 6(4):62-64, 2001. 